\title{
Preparation and Evaluation of Lyophilized Live Attenuated Vaccine of Inclusion Body Hepatitis Hydro-pericardium Syndrome (IBH-HPS) against Challenge in Broiler Chickens
}

\author{
Shahid Ali ${ }^{1}$, M. Shahid Mahmood ${ }^{1}$, Iftikhar Hussain ${ }^{1}$ and M. Nisar Khan ${ }^{2}$ \\ ${ }^{1}$ Institute of Microbiology, University of Agriculture, Faisalabad-38040, Pakistan \\ ${ }^{2}$ Department of Parasitology, University of Agriculture, Faisalabad-38040, Pakistan \\ *For correspondence: drshahiduaf@gmail.com
}

\begin{abstract}
The current study was conducted to prepare and evaluate the efficacy of live attenuated vaccine of IBH-HPS in broiler birds in Pakistan. The IBH-HPS virus was successfully adapted after three blind passages (Vero cell line) and the $4^{\text {th }}$ passage was fully adapted as it gave cytopathogenic effect (CPE) in $48 \mathrm{~h}$ post infection in Vero cells. The $9^{\text {th }}$ passage was determined for attenuation in broiler birds. The attenuated virus was subjected to pathogenecity testing and used as vaccine candidate in broiler chickens. Two hundred and fifty, day-old commercial broiler chicks were purchased from local market, divided into 5 groups and offered feed and water ad libitum. At the age of 15 days, 50 birds in each group were administered with $9^{\text {th }}$ passage live attenuated lyophilized vaccine, the $3^{\text {rd }}$ passage B-propiolactone inactivated vaccine (prepared in the laboratory), oil emulsified inactivated vaccine (UAF-Angavac), commercial vaccine (Bio-Angara, Sana Laboratories, Pakistan), and normal saline, respectively as negative control. The $9^{\text {th }}$ passage live attenuated lyophilized vaccine protected $100 \%$ birds from mortality, clinical signs and high antibody titre was observed. On the other hand, ß-propiolactone inactivated vaccine gave $70 \%$ protection. The oil emulsified inactivated vaccine gave $80 \%$ protection but lower weight gain was observed. The commercial vaccine gave $60 \%$ protection. In negative control $70 \%$ mortality was recorded. The results revealed that the live attenuated lyophilized vaccine showed a higher protection against lethal challenge of IBH-HPS in broilers. C 2015 Friends Science Publishers
\end{abstract}

Keywords: Inclusion body hepatitis hydro pericardium syndrome; Vero cell line; Live attenuated vaccine; Cytopathogenic effect

\section{Introduction}

Avian adenoviruses are known to cause many disease conditions in chickens of all age groups like respiratory infections, hepatitis and problems in egg productivity (Fadly and Winterfield, 1973). Among these manifestations, the egg drop syndrome in layers and Inclusion body hepatitis along with hydropericardium syndrome (IBH-HPS) in broilers (Grgic et al., 2011) are frequently encountered. IBH-HPS is caused by fowl adenovirus serotype- 4 in broiler chickens, which mainly affects age group of 3 to 6 weeks old.

In the last few decades, IBH-HPS has been reported in almost every country around the world (Saifuddin and Wilks, 1991; Goodwin, 1993; Philippe et al., 2005; Gomis et al., 2007). This disease is known as 'Angara Disease' in Pakistan, after its first outbreak at Angara Goth, near Karachi (Akhtar, 1994). In India, the disease was named as 'Leechy disease' or 'Litchi disease' (Manzoor et al., 2013), due to its peculiar appearance of the heart floating in pericardial fluid, which appeared similar to the deshelled leechy (litchi) fruit or IBH-HPS (Abdul-Aziz and Al-Attar,
1991; Balamurugan et al., 2002). In Pakistan the disease was quite destructive and caused the death of 100 million broiler birds in less than two years (Cowen et al., 1996). The disease also caused huge economic losses in South Asian region, owing to mortality, reduced productivity and immune suppression (McFerran and Adair, 2003). Similarly, the disease is widely spread and cause losses to broiler industry in many countries, including India, Iraq, Kuwait, Mexico, Peru, Russia, Japan, Central and South America, Ecuador and Chile (Kataria et al., 2013).

The consistent and predominant gross lesion at necropsy of affected birds is hydropericardium (Anjum et al., 1989). The liver is friable, mottled, swollen and discolored had multiple regions of focal necrosis, pin point or ecchymotic hemorrhages. Microscopic lesions are most commonly seen in the liver, with coagulative necrotic areas and infiltration of mononuclear cells. Histopathological studied revealed intra nuclear inclusion bodies basophilic (INIB) in hepatocytes (Alemnesh et al., 2012). In the bursa of Fabricius, spleen and thymus cyst formation have been observed in some cases (Nakamura et al., 2003).

Good hygiene, bio-security and immunization of the

To cite this paper: Ali, S., M.S. Mahmood, I. Hussain and M.N. Khan, 2015. Preparation and evaluation of lyophilized live attenuated vaccine of inclusion body hepatitis hydro-pericardium syndrome (IBH-HPS) against challenge in broiler chickens. Int. J. Agric. Biol., 17: 658-662 
broiler birds have paramount importance to control this fatal disease. The liver organ formalin inactivated vaccine has been widely used to control IBH-HPS. The autogenously prepared vaccines had impure virus and other pathogens. Moreover, the dose and dosage of these vaccines were not evaluated and mentioned. Therefore, present study was carried out to prepare and evaluate IBH-HPS attenuated vaccine to overcome the losses caused by this devastating disease.

\section{Materials and Methods}

\section{Procurement of IBH-HPS Virus}

IBH-HPS virus was procured from Institute of Microbiology, University of Agriculture, Faisalabad, which was already isolated and purified (Accession number: DQ 264728) (Mansoor et al., 2009). The sequenced virus was fowl adenovirus serotype- 4 .

\section{Cells and Media}

Vero cell line was established in the laboratory from frozen ampoule stored at $-196^{\circ} \mathrm{C}$ imported earlier from the Centre for Applied Microbiology and Research (CAMR), (ECACC 84113001, ECACC, Salisbury, Wilt-shire) (Rasool and Hussain, 2006). Dulbecco's Modified Eagle's Medium (DMEM) with $10 \%$ fetal bovine serum was used as growth medium and with $5 \%$ fetal bovine serum as maintenance medium.

\section{Adaptation and Attenuation of IBH-HPS Virus}

The purified and characterized IBH-HPS virus was taken and a healthy confluent monolayer of Vero cells was infected. Briefly, a total of $0.3 \mathrm{~mL}$ of virus having $10^{5.31}$ $\mathrm{EID}_{50}$ was inoculated and the cell culture flasks were incubated at $37^{\circ} \mathrm{C}$ in the presence of $5 \% \mathrm{CO}_{2}$ in a $\mathrm{CO}_{2}$ incubator and observed for cytopathogenic effects. The culture fluid of each passage of IBH-HPS virus was harvested by three freeze thaw cycles and clarified by centrifugation. The IBH-HPS virus was serially passed on Vero cells for attenuation.

\section{Pathogenicity Testing of IBH-HPS Virus}

Pathogenicity of attenuated IBH-HPS virus was tested in 80 broiler birds of 15 days old, reared in the Animal House of Institute of Microbiology. The total number of broilers were randomly divided into 10 groups $(\mathrm{A}, \mathrm{B}, \mathrm{C}, \mathrm{D}, \mathrm{E}, \mathrm{F}, \mathrm{G}$ and $\mathrm{H})$ each comprising 10 birds. The original virus and passage number 4, 5, 6, 7, 8 and 9 was inoculated in group A to $G$ with dose $0.2 \mathrm{~mL}$ through sub-cutaneous route $(\mathrm{s} / \mathrm{c})$, respectively.

Group $\mathrm{H}$ was inoculated with normal saline as negative control. The birds were examined daily for clinical signs and mortality. At the day 7 and 14 post inoculation, three birds from each group were randomly selected for slaughtering. The gross and pathological lesions on liver, heart and kidneys were observed (Nakamura et al., 1999).

\section{Preparation of Vaccines}

The $9^{\text {th }}$ passage was filtered through $0.2 \mu \mathrm{m}$ filter and lyophilized. The purified IBH-HPS virus was used for the preparation of oil adjuvanted inactivated vaccine using ISA207 montanide oil (SEPPIC, France). The $3^{\text {rd }}$ passage of IBH-HPS virus was taken and inactivated by adding betapropiolactone (Fellows Medical, Mich). The IBH-HPS virus was titrated by calculating plaque forming units $(\mathrm{pfu} / \mathrm{mL})$ and infectivity titre through tissue culture infective dose 50 $\left(\mathrm{TCID}_{50}\right)$. The commercial vaccine (Bio-Angara) was purchased from local market. All the prepared vaccines were tested for sterility on different microbiological media. The stability of the vaccines was also checked before administration to birds (Mansoor et al., 2010).

\section{Evaluation of Vaccines}

The efficacy of different vaccines, including lyophilized live cell culture attenuated, cell culture adapted inactivated, oil adjuvanted inactivated and commercial IBH-HPS vaccines was evaluated through experimental trials. A total number of 250 day old broilers birds were purchased from local market and divided into 5 groups each having 50 birds. Group 1 (G1) was vaccinated with lyophilized live attenuated vaccine through drinking water, group 2 (G2), group 3 (G3) and group 4 (G4) injected subcutaneously with cell culture adapted inactivated vaccine, oil adjuvanted vaccine and commercial vaccine, respectively. Group 5 (G5) with normal saline served as negative control. At 3, 7, 14 and 21 days post vaccination, 5 birds from each group were randomly selected, slaughtered and the organ to body weight ratios were calculated. Gross lesions were recorded on liver, heart and kidneys. The livers were collected and subjected to histopathology (Hussain et al., 2012).

\section{Humoral Immune Response}

The blood samples from five birds in each group were collected at $0,7,14$ and 21 days post-vaccination, before slaughtering, in sterilized test tubes and the serum was separated. The humoral immune response was tested by measuring IBH-HPS antibodies titre in each group by enzyme linked immunosorbent assay (ELISA) using TropBio ELISA kit (Australia). The geometric mean titre was calculated and compared in all vaccinated groups with control negative group.

\section{Cell mediated Immune Response}

The Cell mediated immune response was evaluated in-vivo in all the groups by injecting phyto-haemagglutinin-P (PHA-P) in wattle of the birds. A $100 \mu \mathrm{L}$ of PHA-P was injected intradermally in 5 birds of each group at 14 days post-vaccination, and the swelling of the wattle was 
measured after 24 and $48 \mathrm{~h}$ post-injection with constant tension caliper. The thickness was compared with control negative group (El-Safty et al., 2006).

\section{Challenge Protection Assay}

The remaining birds in each group were challenged with $10^{5}$ embryo infective dose $50\left(\mathrm{EID}_{50}\right)$ of IBH-HPS virus orally at 21 days post-vaccination. Clinical signs, mortality and symptoms were observed twice daily for 21 days.

\section{Statistical Analysis}

Data collected were analyzed through geometric mean titre using descriptive one way analysis of variance (ANOVA) at significance level of $\mathrm{P}<0.05$. The efficacy of all vaccines was compared by Tukey's Test.

\section{Results}

\section{Adaptation of IBH-HPS Virus in Vero Cell Line}

The Vero cell line attained its normal growth in two successive passages after revival, and a healthy monolayer was formed in $36 \mathrm{~h}$ in DMEM. The morphology of normal Vero cells was fibroblast like. The monolayer was intact in passage 1 and passage 2 up to 6 days post infection (P.I.). In the third passage, the changes in monolayer were observed at $96 \mathrm{~h}$ P.I. The complete and clear cytopathogenic effects (CPEs) were observed in passage 4 at $48 \mathrm{~h}$ P.I. The CPEs were clear and consistent up to $9^{\text {th }}$ passage observed at 48 to 72 h P.I.

\section{Pathogenecity Analysis of IBH-HPS Virus}

The pathogenicity of procured IBH-HPS virus reduced considerably after serial passages on Vero cells. The liver to body weight ratio in group treated with passage 9 virus was non-significantly comparable to un-inoculated control group. A maximum hydropericardium was observed in passage 4 group, while it was absent in passage 8 and 9 (Table 1). Hence, the $9^{\text {th }}$ passage was non-pathogenic to broiler birds and attenuated. This passage indicated a TCID50 $(\log 10)$ of $6.70 / \mathrm{mL}$ and plaque forming unit $/ \mathrm{mL}$ $10^{6}(\mathrm{pfu} / \mathrm{mL})$. The $9^{\text {th }}$ passage was lyophilized and its efficacy as live attenuated lyophilized vaccine was evaluated in broilers.

\section{Response of Vaccines in Broilers}

At 14 days of post-vaccination, no apparent untoward reaction was observed in all groups. The results of Mean liver to body weight ratio at 3, 7, 14 and 21 days postvaccination (P.V.) are presented in Table 2.

The liver to body weight ratio was maximum in commercial vaccine immunized group (G4), followed in order by cell culture adapted inactivated vaccine immunized group (G2), oil adjuvanted vaccine immunized group (G3) and lyophilized live attenuated vaccine immunized group (G1). There was negligible difference between control group (G5) and (G1) at $(\mathrm{p}<0.05)$.

Histo-pathologically, no changes in the liver of birds in group 1 were observed as compared to other groups. The changes observed during histopathology of livers of birds in other group included the presence of intra-nuclear inclusion bodies, degenerative changes, infiltration of heterophils, lymphocytes necrosis and atrophy.

For the evaluation of humoral immune response, the results of ELISA antibody titres were observed and geometric mean titres were calculated. The ELISA titre was negligible at day zero (0) in all groups under trial. The overall geomean antibody titre was maximum in G1 followed in order by G3, G2 and G4 (Fig. 1). In contrast, the antibody titre was significantly lower in control group (G5).

The cell mediated immune response was also checked in all the groups under trial of different vaccines. Maximum swelling was observed in group 1 immunized with lyophilized live attenuated vaccine followed in order by group 3 immunized with oil adjuvanted vaccine, group 2 immunized with cell culture adapted inactivated vaccine, group 4 immunized with commercial vaccine.

\section{Protective Efficacy of Vaccines}

The remaining birds in all immunized groups were challenged with virulent IBH-HPS virus through oral route. All the birds in group 1 survived up to 7 days $(100 \%$ efficacy) and had no clinical signs and gross lesions. However, the protective efficacy of cell culture adapted inactivated vaccine $(\mathrm{G} 2)$, oil adjuvanted vaccine (G3) and commercial vaccine (G4) was $70 \%, 80 \%$ and $60 \%$, respectively. The $70 \%$ birds in control group were died after lethal challenge of virulent IBH-HPS virus.

\section{Discussion}

The formalin inactivated liver homogenate is vaccine used extensively in controlling IBH-HPS in broilers (Kumar et al., 1997; Anjum, 1990; Ojkic and Nagy, 2003; Alvarado et al., 2007). This vaccine has many drawbacks, including (1) unpredictable immune response provoked by the vaccine, (2) impurity and the vaccine may contains other viruses and bacteria (Mansoor et al., 2010). The above results revealed that the lyophilized live attenuated vaccine was safer, immunogenic and easy to administer. The live attenuated vaccine provoked a high antibody titre for longer time. The IBH-HPS virus was attenuated on Vero cell line after three blind passages. The $9^{\text {th }}$ passage was completely attenuated determined through pathogenicity testing and used for the preparation of vaccine. The $9^{\text {th }}$ passage was titrated by plaque assay before lyophilization and the virus titre was $10^{6} \mathrm{pfu} / \mathrm{mL}$. The infectivity of the attenuated $9^{\text {th }}$ passage was also checked and gave TCID50 $(\log 10)$ of $6.70 / \mathrm{mL}$. 
Table 1: Evaluation of mean liver to body weight ratio and the development of hydropericardium in experimental broiler birds by different passage number of IBH-HPS virus

\begin{tabular}{lllll}
\hline Group Name & \multicolumn{2}{c}{ Mean liver to body weight ratio } & \multicolumn{2}{c}{ Hydro-pericardium } \\
\cline { 2 - 5 } & 7 days post-inoculation & 14 days post-inoculation & 7 days post-inoculation & 14 days post-inoculation \\
\hline A & 6.3 & 7.5 & ++++ & ++++ \\
B & 6.3 & 7.4 & ++++ & ++++ \\
C & 6.0 & 7.1 & ++++ & +++ \\
D & 6.1 & 6.5 & ++ & ++ \\
E & 5.7 & 6.1 & ++ & - \\
F & 5.2 & 5.6 & - & - \\
G & 4.9 & 5.5 & - & - \\
H & 5.1 & 5.5 & - & ++ \\
\hline
\end{tabular}

Group A, inoculated with $0.2 \mathrm{~mL}$ of purified IBH-HPS virus subcutaneously (s/c); Group B, inoculated with $0.2 \mathrm{~mL}$ passage \# 4of IBH-HPS virus (s/c); Group C, inoculated with $0.2 \mathrm{~mL}$ passage \# 5 of IBH-HPS virus (s/c); Group D, inoculated with $0.2 \mathrm{~mL}$ passage \# 6of IBH-HPS virus (s/c); Group E, inoculated with $0.2 \mathrm{~mL}$ passage \# 7 of IBH-HPS virus (s/c); Group F, inoculated with $0.2 \mathrm{~mL}$ passage \# 8of IBH-HPS virus (s/c); Group G, inoculated with $0.2 \mathrm{~mL}$ passage \# 9 of IBH-HPS virus (s/c); Group H, Negative control.

$++++=10-15 \mathrm{~mL}$ water in pericardial sac, $+++=5-8 \mathrm{~mL},++=3-4 \mathrm{~mL}$ and $-=$ no water

Table 2: Mean liver to body weight ratio \pm SE in different groups vaccinated with four different types of vaccines

\begin{tabular}{llllll}
\hline Age in Days & Group 1 & Group 2 & Group 3 & Group 4 & Group 5 \\
\hline 3 & $5.14 \pm 0.0509^{\mathrm{c}}$ & $5.74 \pm 0.0927^{\mathrm{ab}}$ & $5.56 \pm 0.0927^{\mathrm{b}}$ & $5.960 .0400^{\mathrm{a}}$ & $5.14 \pm 0.0509^{\mathrm{c}}$ \\
7 & $5.48 \pm 0.0663^{\mathrm{c}}$ & $5.96 \pm 0.0509^{\mathrm{b}}$ & $6.14 \pm 0.0509^{\mathrm{b}}$ & $6.84 \pm 0.0812^{\mathrm{a}}$ & $5.64 \pm 0.0927^{\mathrm{c}}$ \\
14 & $5.78 \pm 0.0583^{\mathrm{d}}$ & $6.26 \pm 0.0509^{\mathrm{bc}}$ & $6.480 .1280^{\mathrm{b}}$ & $7.4 \pm 0.0707^{\mathrm{a}}$ & $6 \pm 0.03162^{\mathrm{cd}}$ \\
21 & $6.12 \pm 0.0583^{\mathrm{d}}$ & $6.38 \pm 0.0374^{\mathrm{bc}}$ & $6.5 \pm 0.0316^{\mathrm{b}}$ & $7.9 \pm 0.0707^{\mathrm{a}}$ & $6.2 \pm 0.0707^{\mathrm{dc}}$ \\
\hline
\end{tabular}

(Tukey's HSD alpha<0.05)

Group 1, birds immunized orally with $0.2 \mathrm{~mL}$ of lyophilized live attenuated vaccine; group 2, birds immunized subcutaneously with $0.2 \mathrm{~mL}$ cell culture adapted inactivated; group 3, birds immunized with subcutaneously with $0.2 \mathrm{~mL}$ oil adjuvanted vaccine (UAF-Angavac); Group 4 , birds immunized subcutaneously with $0.2 \mathrm{~mL}$ of commercially formalized liver organ vaccine (SANA Lab, Pakistan); group 5, birds kept as PBS-inoculated control a,b,c,d Mean liver to body weight ratio with different superscripts indicate statistical significance $(\mathrm{p}<0.05)$ between different vaccinated groups

Vero cell line, high infective virus titre coincides well with the appearance of CPEs.

Altogether, the indigenous IBH-HPS virus was adapted and attenuated on Vero cell line (Continuous cell line) without prior passages to embryonated eggs or chicken embryo fibroblasts. Moreover, higher yield of attenuated was also produced on Vero cell line. There was neither any clinical signs nor any death was observed in lyophilized live attenuated vaccine immunized group (G1). The gross lesions score was also zero in case of G1. Mild lesions marked as (1) found in group 3 (G3) and moderate lesion score (2) was observed in cell culture adapted inactivated vaccine group 2 (G2), but a severe lesion score (4) was observed in group 4 (G4) immunized with commercial vaccine. Histo-pathologically, the livers of birds in group 1 had no change as compared to other groups. There were mild to severe changes in the hepatocytes of group 3, group 2 and group 4, respectively. Similar observations were observed by other workers upon experimental trials of cell culture vaccines (Roy et al., 1999). The present study revealed that the live attenuated vaccine can be used for saving the broiler birds from this devastating disease in Pakistan.

\section{Conclusion}

The $9^{\text {th }}$ passage in Vero cell line was non-pathogenic to broiler birds and attenuated. The lyophilized live attenuated

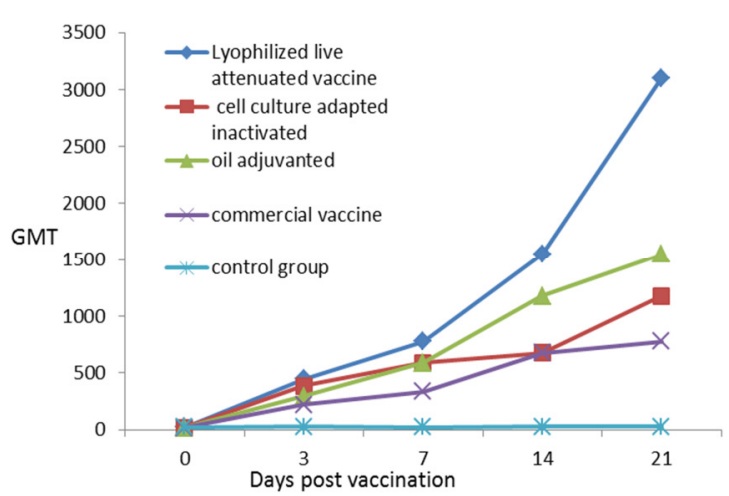

Fig. 1: Geometric mean of ELISA antibody titres (GMT) of different groups of birds immunized with different vaccines

vaccine prepared from the passage 9 significantly induced antibody titres and cell mediated immune responses. The protective efficacy of this vaccine, in terms of mortality, clinical signs and gross lesions, against lethal challenge of virulent IBH-HPS virus was also $100 \%$. However, the field trials in commercial broiler chicks are necessary before recommending this lyophilized live attenuated vaccine for future use against this devastating disease. Above all, the study will pave the way for researchers to work and find out the possibility of the development of oral vaccines for other diseases. 


\section{Acknowledgements}

The research is supported by Higher Education Commission, Islamabad, Pakistan under indigenous scholarship phase VI. The work was also supported by University of Agriculture, Faisalabad, Pakistan, which is highly acknowledged.

\section{References}

Abdul-Aziz, T.A. and M.A. Al-Attar, 1991. New syndrome in Iraqi chicks. Vet. Rec., 129: 272

Akhtar, S., 1994. Hydro-pericardium syndrome in broiler chicken. World's Poult. Sci. J., 50: 177-182

Alemnesh, W., M. Hair-Bejo, I. Aini and A.R. Omar, 2012. Pathogenecity of fowl adenovirus in specific pathogen free chicken embryos. $J$. Comp. Path., 146: 223-229

Alvarado, I.R., P. Villegas, J. El-Attrache, E. Jensen, G. Rosales, F. Perozo and L.B. Purvis, 2007. Genetic characterization, pathogenicity and protection studies with an avian adenovirus isolate associated with inclusion body hepatitis. Avian Dis., 51: 27-32

Anjum, A.D., 1990. Experimental transmission of hydropericardium syndrome and protection against it in commercial broiler chicks. Avian Path., 19: 655-660

Anjum, A.D., M.A. Sabri and Z. Iqbal, 1989. Hydropericarditis syndrome in broiler chickens in Pakistan. Vet. Rec., 124: 247-248

Balamurugan, V., J.M. Kataria, R.S. Kataria, K.C. Verma and T. Nanthakumar, 2002. Characterization of fowl adenovirus serotype-4 associated with hydro-pericardium syndrome in chickens. Comp. Immun. Micro. Inf. Dis., 25: 139-147

Cowen, B.S., H. Lu, D. Weinstock and A.E. Castro, 1996. Pathogenicity studies of fowl adenoviruses isolated in several regions of the world. International symposium on adenovirus infections in poultry, Rauischholzhausen. Germany, pp: 79-88

El-Safty, S.A., U.M. Ali and M.M. Fath, 2006. Immunological parameters and laying performance of naked neck and normallyfeathered genotypes of chicken under winter conditions of Egypt. Int. J. Poul. Sci., 5: 780-785

Fadly, A.M. and R.W. Winterfield, 1973. Isolation and some characteristics of an agent associated with inclusion body hepatitis, hemorrhages, and aplastic anemia in chickens. Avian Dis., 17: 182-193

Gomis, S., L. Babiuk, B. Allan, P. Willson, E. Waters, R. Hecker and A. Potter, 2007. Protection of chickens against a lethal challenge of Escherichia coli by a vaccine containing $\mathrm{CpG}$ oligodeoxynucliotide as an adjuvant. Avian Dis., 51: 78-83

Goodwin, M.A., 1993. Adenovirus inclusion body ventriculitis in chickens and captive bobwhite quail (Colinus virginianus). Avian Dis., 37: $568-571$

Grgic, H., D.H. Yang and E. Nagy, 2011. Pathogenicity and complete genome sequence of a fowl adenovirus serotype 8 isolate. Virus Res., 156: $91-97$
Hussain, I., M.S. Mahmood, M.I. Arshad, M. Akhtar, F. Mahmood and A. Rafique, 2012. Immune system dysfunction in broiler chicken experimentally inoculated with fowl adenovirus serotype 4 associated with inclusion body hepatitis hydropericardium syndrome. Turk. J. Vet. Anim. Sci., 36: 223-230

Kataria, J.M., K. Dhama, S. Nagarajan, S. Chakraborty, A. Kaushal and R. Deb, 2013. Fowl adenoviruses causing hydropericardium syndrome in poultry. Adv. Ani. Vet. Sci., 1: 5-13

Kumar, R., R. Chandra, S.K. Shukla, D.K. Agrawal and M. Kumar, 1997. Hydropericardium syndrome in India: a preliminary study on causative agent and control of disease by inactivated autogenous vaccines. Trop. Anim. Heal. Prod., 29: 158-164

Mansoor, M.K., I. Hussain, M. Arshad and G. Muhammad, 2010. Preparation and evaluation of chicken embryo adapted fowl adenovirus serotype-4 vaccine in broiler chickens. Trop. Anim. Heal. Prod., 43: 331-338

Mansoor, M.K., I. Hussain, M. Arshad, G. Muhammad, M.H. Hussain and M.S. Mehmood, 2009. Molecular characterization of fowl adenovirus serotype 4 (FAV- 4) isolate associated with fowl hydropericardium-hepatitis syndrome in Pakistan. Pak. J. Zool., 41: 269-276

Manzoor, S., Z. Hussain, S.U. Rahman and I. Hussain, 2013. Identification of antibodies against hydropericardium syndrome in wild birds. Brit. Poult. Sci., 54: 325-328

McFerran, J.B. and B.M. Adair, 2003. Hydropericardium Syndrome. In: Diseases of Poultry, $11^{\text {th }}$ edition, pp: 220-221. Saif, Y.M., J.H Barnes, J.R. Glisson, A.M. Fadly, L.R. McDougald and D.E. Swayne (eds.). Iowa State University Press, Ames, Iowa, USA

Nakamura, K., M. Mase, S. Yamaguchi, T. Shiobahara and N. Yuasa, 1999. Pathologic study of specific pathogen free chicks and hens inoculated with adenovirus isolated from hydropericardium syndrome. Avian Dis., 43: 414-423

Nakamura, K., T. Shoyama, M. Mase, T. Imada and M. Yamada, 2003. Reproduction of hydropericardium syndrome in three-week-old cyclophosphamide treated specific pathogen free chickens by adenoviruses from inclusion body hepatitis. Avian Dis., 47: 169-174

Ojkic, D. and E. Nagy, 2003. Antibody response and virus distribution in chickens inoculated with wild-type and recombinant fowl adenovirus. Vaccine, 22: 42-48

Philippe, C., H. Grgic and E. Nagy, 2005. Inclusion body hepatitis in young broiler breeders associated with a serotype 2 adenovirus in Ontario, Canada. J. Appl. Poult. Res., 14: 588-593

Rasool, M.H. and I. Hussain, 2006. Preparation and evaluation of Vero cell infectious bursal disease vaccine in Pakistan. Vaccine, 24 2810-2814

Roy, P., A. Kotteeswaran and R. Manickam, 1999. Efficacy of an inactivated oil emulsion vaccine against hydropericardium syndrome in broilers. Vet. Rec., 145: 458-459

Saifuddin, M. and C.R. Wilks, 1991. Vertical transmission of adenovirus associated with inclusion body hepatitis. $N$. Z. Vet. J., 39: 50-52

(Received 05 July 2014; Accepted 22 July 2014) 